\title{
Balanço de carbono em cidades da Amazônia: estudo de caso em Belém, Brasil
}

Carbon balance in Amazon cities: case of study in Belém, Brazil

Matheus Paes de Assumpção[a], Paulo Amador Tavares[a] (i), Eliane de Castro Coutinho[a] (1)

[a] Universidade do Estado do Pará (UEPA), Belém, PA, Brasil

Como citar: Assumpção, M. P., Tavares, P. A., \& Coutinho, E. C. (2019). Balanço de carbono em cidades da Amazônia: estudo de caso em Belém, Brasil. urbe. Revista Brasileira de Gestão Urbana, 11, e20180186. https://doi.org/10.1590/21753369.011.e20180186

\section{Resumo}

O município de Belém, no Pará, tem apresentado crescimento contínuo de sua área urbanizada e da frota de veículos nas últimas décadas. Essas mudanças estão também associadas a reduções na área de cobertura vegetal e ao aumento das emissões de $\mathrm{CO}_{2}$ locais. Há, portanto, a necessidade de se entender, de maneira integrada, a dinâmica das emissões regionais de gases de efeito estufa (GEE) em áreas urbanas. 0 estudo objetivou a elaboração de um balanço de carbono entre as emissões pelo transporte público e o potencial de captura pela vegetação no município de Belém. A emissão do transporte público foi estimada por meio do dimensionamento dos percursos das rotas de ônibus, e a captura, por sua vez, por intermédio de classificação semiautomática de imagens de satélite de alta resolução e referências bibliográficas. Identificou-se um potencial de captura equivalente a 5.117,82 toneladas de carbono/ano, enquanto a emissão pelo transporte público correspondeu a 146.889,61 toneladas de $\mathrm{CO}_{2} /$ ano. Assim, a captura de carbono pela vegetação representou 3,48\% do total de $\mathrm{CO}_{2}$ emitido pelo sistema de transporte público. Por fim, foi possível notar a necessidade de implantação de políticas integradas para obtenção de resultados mais expressivos na mitigação de emissões de $\mathrm{CO}_{2}$ em Belém.

Palavras-chave: Sequestro e estoque de carbono. Áreas urbanas na Amazônia. Cidades sustentáveis.

\section{Abstract}

The municipality of Belém, Brazil, is presenting continuous growth of its urbanized area and its vehicle fleet in recent decades. These changes are also associated with reductions in tree coverage areas and have increased local $\mathrm{CO}_{2}$ emission. Henceforth, there is a need to understand the dynamics of regional greenhouse gases (GHG) emission in an integrated manner. The study aimed to elaborate a carbon balance between the emission from public transportation and sequestration by urban vegetation in the municipality of Belém. Thus, carbon balance was performed by dimensioning bus routes and the carbon sequestration and also estimated through a semiautomatic classification of high-resolution satellite images and literature references. Thus, there was a sequestration potential of 5.117,82 tons of carbon per year, while public transportation busses' emissions corresponded to 146.889,61 tons of carbon/year.

MPA é bacharel em Engenharia Ambiental, e-mail: matheusump@gmail.com

PAT é mestre em Ciências Ambientais, bacharel em Engenharia Ambiental, e-mail: atavares.paulo@gmail.com

ECC é doutora em Ciências Ambientais, graduada em Meteorologia, e-mail: elianerik@gmail.com 
Thereby, carbon sequestration accounted for $3.48 \%$ of $\mathrm{CO}_{2}$ emission from the public transportation system. Lastly, it was possible to notice the need to implement integrated policies to acquire higher values of $\mathrm{CO}_{2}$ emissions' mitigation in the municipality of Belém.

Keywords: Carbon sequestration and stock. Amazon urban areas. Sustainable cities.

\section{Introdução}

As emissões de $\mathrm{CO}_{2}$ no Brasil, assim como em outros países em desenvolvimento, apresentam perfil distinto dos países desenvolvidos. Mesmo com a forte urbanização e industrialização do país no final do século XX (Santos, 2005), ainda prevalecem emissões por processos relacionados a mudanças no uso do solo. No entanto, enquanto as taxas de desmatamento anuais na Amazônia apresentaram reduções mais recentemente, as cidades brasileiras se expandem, intensificando processos que contribuem para as mudanças climáticas globais (Brasil, 2017; Maricato, 2013).

Nesse sentido, constata-se que as cidades têm se configurado como principais centros de emissão de $\mathrm{CO}_{2}$ no mundo, e, por esse motivo, uma série de iniciativas e inovações tem sido adotada na esfera local em diferentes países para se mitigar emissões de gases de efeito estufa (GEE) (Lee \& Erickson, 2017; Broto \& Bulkeley, 2013; Satterthwaite, 2008). No Brasil, enfatiza-se, no entanto, a existência de obstáculos para a implantação de políticas ambientais nessa esfera, como a falta de conhecimentos científicos adequados para diferentes municípios (Frey, 2009).

0 presente estudo, portanto, é realizado em Belém, no Pará, e busca contribuir para o entendimento integrado das emissões regionais na Amazônia, com ênfase nas emissões urbanas de $\mathrm{CO}_{2}$ na Região Amazônica. 0 município de Belém tem apresentado crescimento contínuo e significativo nas últimas décadas. Sendo uma das principais metrópoles da Amazônia, a cidade, ao mesmo tempo que se expande em termos populacionais e territoriais, tem gerado reduções de suas áreas de cobertura vegetal e um aumento na frota de veículos à base de combustíveis fósseis, processos que contribuem para o aumento das emissões de GEE (Rodrigues, 2013; Amaral et al., 2009).

Dessa forma, o estudo é constituído de uma revisão bibliográfica (que apresenta tópicos referentes a questões estruturais pertinentes à temática do estudo), com descrição da metodologia empregada, apresentação dos resultados obtidos por meio das análises realizadas e das principais discussões e sugestões pertinentes ao avanço do debate sobre a dinâmica de $\mathrm{CO}_{2}$ no município de Belém.

Assim, o objetivo geral desta pesquisa foi elaborar um balanço de $\mathrm{CO}_{2}$ entre o transporte público e a vegetação urbana no município de Belém. Isso foi alcançado a partir da quantificação dos GEE emitidos pelo transporte público local, da estimativa do potencial de captura de carbono pela vegetação urbana e da comparação dessas duas variáveis.

\section{Revisão de literatura}

\section{Emissões de $\mathrm{CO}_{2}$ e mudanças climáticas}

No fim do século XX, a dinâmica das mudanças climáticas passou a gerar preocupações por conta de uma mudança substancial no caráter do $\mathrm{CO}_{2}$ presente na atmosfera. Nesse período, estudos indicaram a interferência antrópica inédita nos teores de $\mathrm{CO}_{2}$ atmosférico. No âmbito dessas contribuições, as queimas de combustíveis fósseis e as atividades industriais foram responsáveis, segundo estimativas, por 78\% do aumento nas emissões de $\mathrm{CO}_{2}$ entre 1970 e 2010 (Pachauri \& Meyer, 2014; Silveira et al., 2008).

Nesse sentido, entre os principais setores econômicos contribuintes para as emissões de $\mathrm{CO}_{2}$ nos anos mais recentes, ressaltam-se: i) a geração de eletricidade e aquecimento (25-26\%); ii) a indústria (19-21\%); iii) a silvicultura (17\%); iv) a agricultura (14\%); e v) os transportes (13-14\%) (Pachauri \& Meyer, 2014; 
Dodman, 2009). Dentro do setor de transportes, $73 \%$ das emissões do $\mathrm{CO}_{2}$ são atribuídas aos transportes rodoviários (D'Agosto, 2015).

No Brasil, há predominância de emissões vinculadas a mudanças no uso do solo (55\% do total) e a atividades agrícolas (25\% do total), enquanto o setor de produção de energia contribui com $17 \%$ com essas emissões. Assim, ressalta-se a importância do Estado do Pará, que tem liderado o ranking de desmatamento há 10 anos entre os Estados da Amazônia Legal (sendo responsável por 44\% do desmatamento ocorrido na região nesse período) (Brasil, 2017; Nobre, 2008).

Analogamente, destaca-se a existência de paradoxos nas raízes da estrutura econômica vigente na Amazônia. Ao mesmo tempo que setores-chave responsáveis pelo desenvolvimento da região contribuem substancialmente para as emissões nacionais de $\mathrm{CO}_{2}$, eles são vulneráveis aos impactos ocasionados pelas mudanças climáticas globais. Essa vulnerabilidade se encontra associada, principalmente, à dependência de disponibilidade e equilíbrio de recursos naturais para os setores econômicos, além da possibilidade de aumento de eventos extremos na região - como incêndios florestais e comprometimentos das cadeias produtivas de alimentos (Pachauri \& Meyer, 2014; Rivero et al., 2009; Fearnside, 2009; Andrade \& Costa, 2008).

Nos espaços urbanos, por sua vez, as emissões vinculadas aos transportes tendem a apresentar maior destaque, apesar de existirem variações entre diferentes cidades. No Brasil, São Paulo e Rio de Janeiro, por exemplo, apresentam, respectivamente, $59,7 \%$ e 29,7 das emissões vinculadas aos transportes, $23,6 \%$ e 36,5\% relacionadas à geração de resíduos e 9,7\% e $6 \%$ referentes a processos industriais (Dodman, 2009). Quanto às emissões geradas diretamente no espaço urbano, o setor de transportes é responsável por mais de 90\% da emissão de alguns gases, como CO e NOX (Alonso et al., 2010).

Em termos de $\mathrm{CO}_{2}$, os ônibus contribuem com uma média de $1,04 \mathrm{~kg} \mathrm{de} \mathrm{CO}_{2} / \mathrm{km}$, enquanto automóveis individuais e motocicletas, com 0,15 e 0,06 $\mathrm{kg} \mathrm{de} \mathrm{CO}_{2} / \mathrm{km}$, respectivamente (Carvalho, 2011). De tal forma que se é perceptível a necessidade de estudos que analisem esses tipos de emissões em grandes centros urbanos e como é a resiliência urbana a essas emissões locais, é também fundamental a investigação dos impactos da urbanização em termos de regulação climática local.

\section{Urbanização desordenada}

As cidades configuram-se como os principais polos de emissão de gases de efeito estufa (GEE) do planeta (Satterthwaite, 2008). Nas últimas décadas, as nações em desenvolvimento apresentaram padrões mais acentuados de crescimento populacional e de desenvolvimento de aglomerados urbanos, quando comparadas com países desenvolvidos, no mesmo período (Heilig, 2012). No Brasil, esse crescimento das cidades teve forte influência do processo de industrialização da economia do país, o que permitiu a manutenção de um crescimento linear das populações urbanas (Santos, 2005).

De maneira integrada, portanto, esses fatores estruturaram um modelo de desenvolvimento das cidades pautado na horizontalização de seu espaço. Uma das características desse processo é o surgimento de novas áreas de moradia apresentando diversos problemas, como segregação social, dependência de automóveis, entre outros. Entre as capitais brasileiras, a periferia de Belém foi a que apresentou maior crescimento entre 1991 e 1996, com 157,9\%, enquanto a de Curitiba cresceu 28,2\% (Maricato, 2013; Cardoso \& Ventura, 2013; Farr, 2011). Por isso, ressalta-se a importância de estudos que considerem a área urbana desse município.

É esse cenário que permite o avanço da dominância dos veículos motorizados sobre o território da Região Metropolitana de Belém. Para o período entre 2001 e 2012, o município apresentou o sétimo maior aumento no número de automóveis (117\%) e o maior aumento no número de motocicletas entre as capitais brasileiras (838\%) (Rodrigues, 2013). Associada a essa dinâmica estão os altos índices de emissão de poluentes e gases de efeito estufa vinculados aos transportes nos espaços urbanos, como previamente apontados. 
Ainda intrínseco ao fenômeno de expansão das metrópoles, ocorre um comprometimento de áreas vegetadas nelas. Para o município de Belém, estima-se que, até 1986, foram desmatados em torno de $50 \%$ de seu território $\left(597,5 \mathrm{~km}^{2}\right)$ e que, posteriormente, entre 1986 e 2006, foram perdidos mais $211,2 \mathrm{~km}^{2}$ de vegetação (Amaral et al., 2009). No ano de 2017, considerando-se a área do município de Belém (sem massa d'água, mas com ilhas e área periurbana), identificou-se $\sim 67 \%$ de cobertura vegetal (vegetação urbana e primária), enquanto, para a área densamente povoada dos distritos administrativos, esse valor de cobertura vegetal caiu para $\sim 37 \%$ (Tavares et al., 2019). A percepção dessa reduzida cobertura vegetal nas áreas com maior contingente populacional humano torna relevante as análises da interação da vegetação com a climatologia local.

Para as áreas urbanas, ressalta-se a importância da vegetação urbana por meio da geração de diferentes benefícios, como a mitigação de poluição sonora e da influência das chuvas para enchentes, a melhoria da qualidade do ar e a redução de temperaturas, assim como benefícios que transcendem a escala local, como a mitigação de mudanças climáticas por intermédio do processo de captura de $\mathrm{CO}_{2}$ (Carvalho \& Szlafsztein, 2019; Tyrväinen et al., 2014; Roy et al., 2012; Dimoudi \& Nikolopoulou, 2003; Nowak \& Crane, 2002). Assim, destaca-se a captura de GEE, uma vez que esses gases são os mais produzidos no ambiente urbano em razão do processo de urbanização e de ocupação desordenada do solo.

\section{Captura de GEE}

A produtividade primária das florestas consiste no processo de crescimento da sua vegetação por meio da incorporação de compostos vitais. Dentro desse processo, mediante a realização de fotossíntese, há a incorporação de carbono atmosférico na biomassa vegetal (raízes, tronco e folhas) e fixação dele no solo, constituindo o processo de captura de carbono (Luyssaert et al., 2008; Ohtsuka et al., 2007; Montagnini \& Nair, 2004). Entre os compartimentos de armazenamento de carbono na biomassa, enfatizam-se a importância e a dominância do caule, por ser mais perene, volumoso e isento de perdas de biomassa frequentes para o meio externo (Malhi et al., 2004).

Dessa forma, a vegetação de ecossistemas florestais, os solos, os combustíveis fósseis e os oceanos são compreendidos como os principais sistemas de armazenamento de carbono do planeta, com, respectivamente, 560, 1.515, 6.500 e 36.000 bilhões de toneladas de carbono. A atmosfera, que armazena 730 bilhões de toneladas, por sua vez, é considerada um sistema transitório, visto que seu carbono é transferido para os sistemas oceânicos e a biomassa. Os desequilíbrios no teor de $\mathrm{CO}_{2}$ atmosférico são, portanto, relacionados, principalmente, ao consumo dos estoques dos sistemas de combustíveis fósseis e florestais (Montagnini \& Nair, 2004; Pacheco \& Helene, 1990; Houghton \& Woodwell, 1989).

Dos diferentes ecossistemas, as florestas tropicais apresentam altas taxas de produtividade primária, sobretudo pela temperatura, pela umidade e pelo grau de luminosidade intrínsecos a esse tipo de floresta (Eggleston et al., 2006; Pacheco \& Helene, 1990). Apresentando grande diversidade de espécies (que variam entre 20 e $50 \mathrm{~m}$ de altura), temperaturas anuais na média de $25{ }^{\circ} \mathrm{C}$ e alta pluviosidade no ano, essas florestas se constituem, dessa forma, como uma parcela importante da produtividade total dos ecossistemas terrestres (Poorter et al., 2015; IBGE, 2012; Luyssaert et al., 2008).

Em virtude do potencial de captura e armazenamento de carbono por esses ecossistemas, maior ênfase passou a ser dada aos processos de funcionamento e às inter-relações vinculadas a esse processo, com o surgimento de estudos majoritariamente empíricos (Coskun et al., 2016; Poorter et al., 2015; Woodbury et al., 2007; Malhi et al., 2004; West \& Marland, 2002). Para os ecossistemas florestais, dois métodos principais são utilizados: os diretos, que consistem em amostragens periódicas nos ecossistemas florestais em determinada área; e os indiretos, que usam dados de amostragens diretas para geração de modelos matemáticos de previsão (Silveira et al., 2008). Esses modelos indiretos, geralmente, fundamentam-se em dados previamente extraídos em campo, com processamentos estimativos derivados de outras fontes de dados (como o sensoriamento remoto). 
Para a aplicação de ambos os métodos, no entanto, existem obstáculos, principalmente em se tratando de ambientes florestais tropicais. Um desses problemas consiste na não padronização e na falta de clareza das metodologias de mensuração entre diferentes estudos (Rosa et al., 2014). Outro obstáculo consiste na complexidade das florestas tropicais, em que a variedade de espécies, os problemas de acessibilidade, a vasta extensão das áreas florestais, entre outros fatores, dificultam a geração e a exatidão de dados (Fearnside, 1997). Dessa forma, compreende-se a necessidade do desenvolvimento de dados com maior confiabilidade temática que possam ser aplicados com mais precisão localmente.

Para a Floresta Amazônica, diferentes valores de captura de carbono são encontrados na literatura, enfatizando a heterogeneidade dela. Alguns trabalhos apresentam (valores dados em toneladas/ha/ano): 0,97 em áreas de Rondônia e de Manaus (Phillips et al., 1998), 1,2 em Manaus (Higuchi et al., 2004), 1,9 em Rondônia (considerando a biomassa acima e abaixo do solo) (Grace et al., 1995), 2,55 para a região nordeste do Pará e 3,1 como média geral para a América Latina (Malhi et al., 2004).

A compreensão desses valores pode ser vista dentro de uma perspectiva de políticas públicas para o desenvolvimento sustentável local, uma vez que a existência dessa regulação climática local está intrinsicamente vinculada com a qualidade de vida humana (Van Oudenhoven et al., 2018). Assim, entende-se que o desenvolvimento de estudos relacionados com o sequestro e o estoque de carbono em áreas urbanas pode contribuir para um planejamento urbano sustentável.

\section{Planejamento urbano sustentável}

Recentemente, conceitos e estudos, como os de metabolismo urbano e metabolismo de carbono (Guo et al., 2017; Weisz \& Steinberger, 2010; Kennedy et al., 2007), têm surgido nas décadas recentes e também influenciado vertentes de planejamento urbano sustentável, como as Ecocidades, as Cidades Inteligentes, as Cidades de Baixo Carbono, as Cidades de Zero Carbono, entre outras (Tan et al., 2017; Su et al., 2016).

Podem ser ressaltadas algumas políticas de mitigação de emissões atualmente adotadas pelas cidades: i) melhorias na infraestrutura urbana (como as mudanças na matriz energética); ii) maior eficiência dos ambientes construídos; iii) moldagens da forma urbana (como o adensamento populacional); iv) ampliação da captura de carbono urbano via reflorestamento; e v) intervenções no tráfego urbano (como o incentivo a modais de baixa emissão de carbono e restrições a automóveis). Considerando algumas dessas vertentes, identificou-se o prevalecimento de intervenções na infraestrutura urbana (31,1\%), nos ambientes construídos $(24,7 \%)$ e nos transportes $(18,8 \%)$, enquanto medidas de captura corresponderam a apenas 5\% das intervenções (Lee \& Erickson, 2017; Broto \& Bulkeley, 2013).

Assim, percebe-se a importância do desenvolvimento de estudos que compreendam o ciclo do carbono em uma escala local, de tal forma que políticas públicas, vinculadas à infraestrutura verde urbana, possam ser desenvolvidas a ponto de aprimorar o bem-estar social em termos de redução dos impactos da emissão de GEE.

\section{Materiais e métodos}

\section{Área de estudo}

0 município de Belém apresenta uma área territorial total equivalente a 1.059,458 km² (IBGE, 2010), constituída de oito distritos administrativos (distritos de Belém, Guamá, Entroncamento, Sacramenta, Bengui, Icoaraci, Outeiro e Mosqueiro), algumas ilhas nos seus arredores e de áreas hidrográficas (Belém, 2012). No entanto, para as análises realizadas neste estudo, considerou-se uma área terrestre equivalente a $264,40 \mathrm{~km}^{2}$, desconsiderando áreas isoladas dos fluxos de ônibus do município (assim como o distrito de Mosqueiro e o arquipélago de ilhas). A Figura 1 ilustra a área considerada dentro da área total do município. 


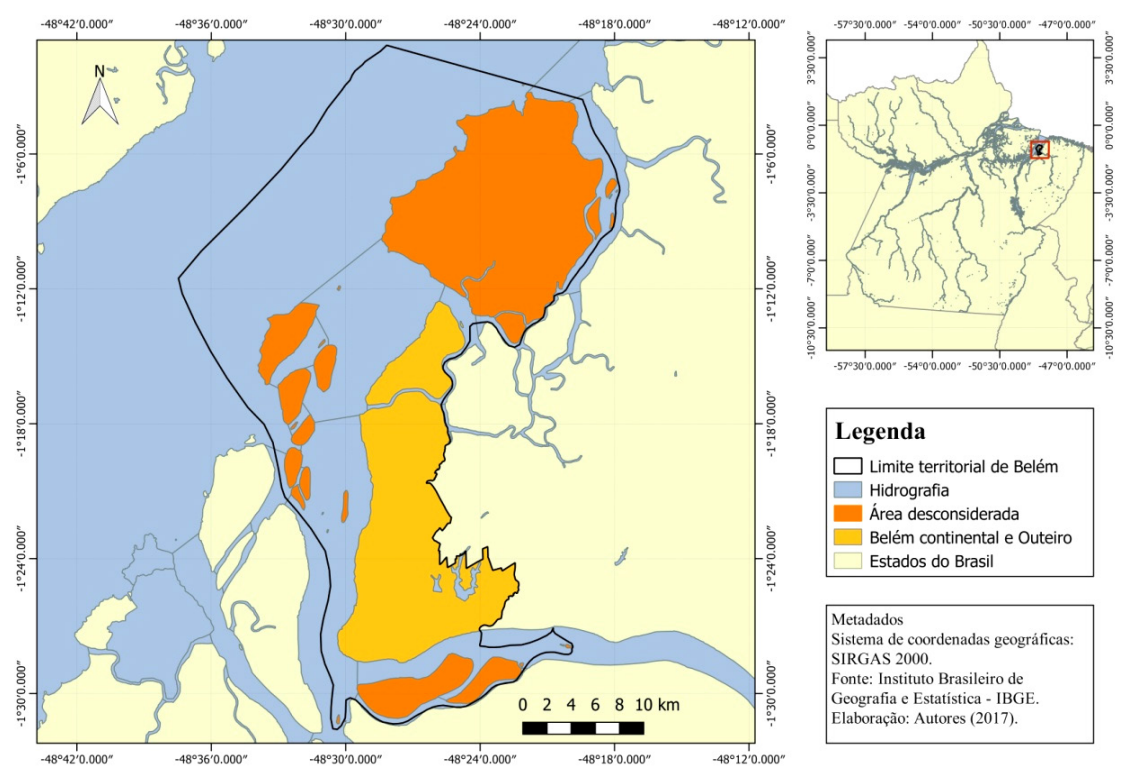

Figura 1 - Mapa de localização destacando as áreas consideradas e descartadas no estudo.

Fonte: Autores (2018).

O município apresentava ainda uma população estimada de 1.452 .275 para o ano de 2017, representando uma densidade demográfica de 1.315,26 habitantes por $\mathrm{km}^{2}$, além de uma frota de veículos equivalente a 414.678 para o ano de 2015 (constituída de 289.537 veículos individuais, 119.249 motocicletas diversas, 3.806 ônibus e 2.086 micro-ônibus) (IBGE, 2010). Os fragmentos florestais do município são classificados como florestas de terra firme e florestas de várzea, sendo subtipos das florestas ombrófilas densas (Amaral et al., 2009).

\section{Coleta de dados}

Para a realização do estudo, foram utilizados quatro principais tipos de insumo: dados provenientes de bases de dados de órgãos públicos, imagens de satélites de alta resolução de bases de dados públicos, bases georreferenciadas e softwares para processamento desses dados. 0 Quadro 1 lista e descreve os insumos utilizados.

Quadro 1 - Listagem e descrição de insumos utilizados na pesquisa

\begin{tabular}{|c|c|c|c|}
\hline $\mathbf{N}^{\circ}$ & Insumo & Obtenção de dados & Descrição \\
\hline 1 & $\begin{array}{c}\text { Dados de rota de linhas de } \\
\text { ônibus. }\end{array}$ & $\begin{array}{c}\text { Superintendência Executiva } \\
\text { de Mobilidade Urbana de } \\
\text { Belém (SEMOB) }\end{array}$ & $\begin{array}{l}\text { Referentes ao ano de } 2016 \text { para } 178 \text { linhas } \\
\text { de ônibus operantes na Região } \\
\text { Metropolitana de Belém (RMB). }\end{array}$ \\
\hline 2 & $\begin{array}{l}\text { Dados de número de } \\
\text { viagens contratadas. }\end{array}$ & SEMOB & $\begin{array}{c}\text { Referentes ao ano de } 2016 \text { para as } 20 \\
\text { empresas operantes de linhas de ônibus na } \\
\text { RMB. }\end{array}$ \\
\hline 3 & Software QGIS. & --- & Versão 2.18 .17 \\
\hline 4 & Software MapSource. & --- & $\begin{array}{l}\text { Versão 6.16.3, sendo utilizada base } \\
\text { georreferenciada de arquivo pessoal. }\end{array}$ \\
\hline 5 & Microsoft Excel. & --- & Versão 12.0.6776.5000. \\
\hline 6 & $\begin{array}{l}17 \text { imagens de satélite do } \\
\text { sistema Planet Labs Inc. }\end{array}$ & $\begin{array}{l}\text { Secretaria de Estado de } \\
\text { Meio Ambiente e } \\
\text { Sustentabilidade (SEMAS-PA) }\end{array}$ & $\begin{array}{c}\text { Referentes ao dia } 28 \text { de julho de } 2017, \text { com } \\
\text { resolução espacial de } 3 \mathrm{~m} .\end{array}$ \\
\hline 7 & $\begin{array}{l}\text { Base digital referenciada do } \\
\text { município. }\end{array}$ & 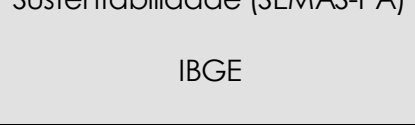 & $\begin{array}{l}\text { Contendo sistema viário, hidrografia, } \\
\text { divisão de distritos e limites municipais do } \\
\text { Estado. }\end{array}$ \\
\hline
\end{tabular}

Fonte: Autores (2018). 


\section{Análise de dados}

\section{Emissão por transporte público}

Realizou-se uma sistematização dos dados referentes às rotas das 178 linhas de ônibus disponibilizadas pela SEMOB utilizando o software MapSource. A partir dessa sistematização, foram obtidas as distâncias percorridas pelas linhas dentro da área considerada neste estudo. Dessa forma, para as linhas operantes na RMB com início de viagem em outro município, foi desconsiderada a distância percorrida fora do território de Belém. Outros fatores específicos da frota de ônibus operante (como idade, desgaste ou eficiência dos mecanismos de mitigação da poluição emitida) também não foram considerados.

De posse, portanto, das distâncias percorridas, esses dados foram correlacionados com as empresas operantes das linhas. Assim, para cada uma das 20 empresas operantes de viagens na RMB, calculou-se a média das distâncias das linhas operadas, e, posteriormente, essa média foi associada ao número de viagens anuais executadas pelas empresas. Dessa forma, foi possível obter a distância média total anual percorrida por todas as linhas de ônibus no município de Belém para o período de 2016.

Em seguida, realizou-se a conversão da distância anual percorrida pelos ônibus para massa de $\mathrm{CO}_{2}$ emitida por eles. Para isso, considerou-se o óleo diesel como combustível básico e aplicou-se uma taxa de conversão (de km para $\mathrm{kg}$ de $\mathrm{CO}_{2}$ ) equivalente a 1,04 (Carvalho, 2011). A taxa de conversão foi adaptada a este estudo, visando considerar apenas a emissão in loco no momento de queima do combustível (desconsiderando, portanto, outras etapas do ciclo de produção do óleo diesel).

\section{Captura de carbono}

Após a obtenção das 17 imagens de satélite, produziu-se, por meio do uso do software QGIS 2.18.17 Las Palmas, um mosaico de imagens com toda a área territorial de Belém. Nesse mesmo software, extraiu-se somente a área considerada no estudo, ou seja, excluíram-se as áreas que não faziam parte da rota dos ônibus. Em seguida, aplicou-se a ferramenta de classificação semiautomática não supervisionada de imagens (SCP) para identificação das tipologias de cobertura de solo do município (Vale \& Balieiro, 2013; Rozenstein \& Karnieli, 2011). Ressalta-se que foram utilizadas imagens de alta qualidade para permitir a identificação de espécimes de árvores individuais da arborização urbana.

Nessa classificação, além da cobertura vegetal desejada, foram identificadas outras três categorias de uso de solo. A divisão de categorias (principalmente as de vegetação) foi realizada com base nas características espectrais dos elementos que as compõem (Quadro 2) e na capacidade do software de identificar e agrupar esses elementos em grupos. Para cada uma dessas categorias, uma quantidade específica de pontos amostrais foi aplicada, visando prevenir equívocos na análise e enfatizando a própria importância do dado.

Quadro 2 - Tipologias de classificação de solo da classificação semiautomática

\begin{tabular}{cccc}
\hline Código & Tipologia & \multicolumn{1}{c}{ Descrição } & $\begin{array}{c}\mathbf{N}^{\circ} \text { de regiões de } \\
\text { interesse (ROI) }\end{array}$ \\
\hline 1 & Vegetação densa & $\begin{array}{l}\text { Fragmentos florestais e árvores individuais de } \\
\text { médio/grande porte. }\end{array}$ & 2.000 \\
2 & $\begin{array}{c}\text { Vegetação } \\
\text { rasteira }\end{array}$ & $\begin{array}{l}\text { Áreas de gramado e de cobertura vegetal de } \\
\text { pequeno porte. } \\
\text { Corpos hídricos (lagos, rios e segmentos da Baía do } \\
\text { Guajará). } \\
\text { Ambiente construído, composto de diferentes tipos } \\
\text { de edificação e de vias asfaltadas. }\end{array}$ & 1.600 \\
4 & Hidrografia & Impermeável & 2.300 \\
\hline
\end{tabular}

Fonte: Autores (2018). 
A partir do arquivo gerado contendo as tipologias de classificação do solo, foram aplicadas as bases georreferenciadas para a construção de mapas relevantes. O Quadro 2 apresenta, portanto, as classificações criadas, assim como sua descrição e seu número de pontos amostrais empregados.

Com base na identificação da área de cobertura vegetal do município e na tipologia da vegetação local, foi utilizada referência da literatura para determinação do potencial de captura de carbono, além da biomassa florestal. A escolha dessas taxas de conversão considerou recomendações do documento de Diretrizes para Inventário Nacional de Gases de Efeito Estufa do IPCC (selecionando-se, portanto, dados de especificidade regional para cálculo) (Eggleston et al., 2006). Assim, aplicou-se um coeficiente equivalente a 2,55 toneladas anuais de incremento de matéria seca por hectare de vegetação densa, fazendo referência a florestas tropicais úmidas da região nordeste do Estado do Pará (Malhi et al., 2004).

Por fim, para a transformação do incremento de matéria seca de vegetação em massa de carbono, foram também utilizadas referências do IPCC, aplicando-se um coeficiente de conversão de 0,49 (Eggleston et al., 2006). No entanto, ressalta-se que as referências utilizadas se limitam a considerar o incremento de carbono na parte superficial da vegetação, desconsiderando, portanto, as parcelas de carbono incorporadas em componentes mortos e subterrâneos da biomassa.

\section{Balanço geral de carbono}

Para construção do balanço geral de carbono, foram utilizados os resultados das análises de emissão pelo transporte público e da captura de carbono. Assim, o balanço de carbono se deu a partir da subtração da emissão pelos ônibus do sistema de transporte público do município e do potencial de captura anual de carbono pela sua área vegetada. A Figura 2 apresenta um fluxograma resumido de procedimentos e etapas que se sucederam para obtenção do balanço de carbono, enquanto o Quadro 3 sintetiza e descreve as fórmulas empregadas nos diferentes processos executados.

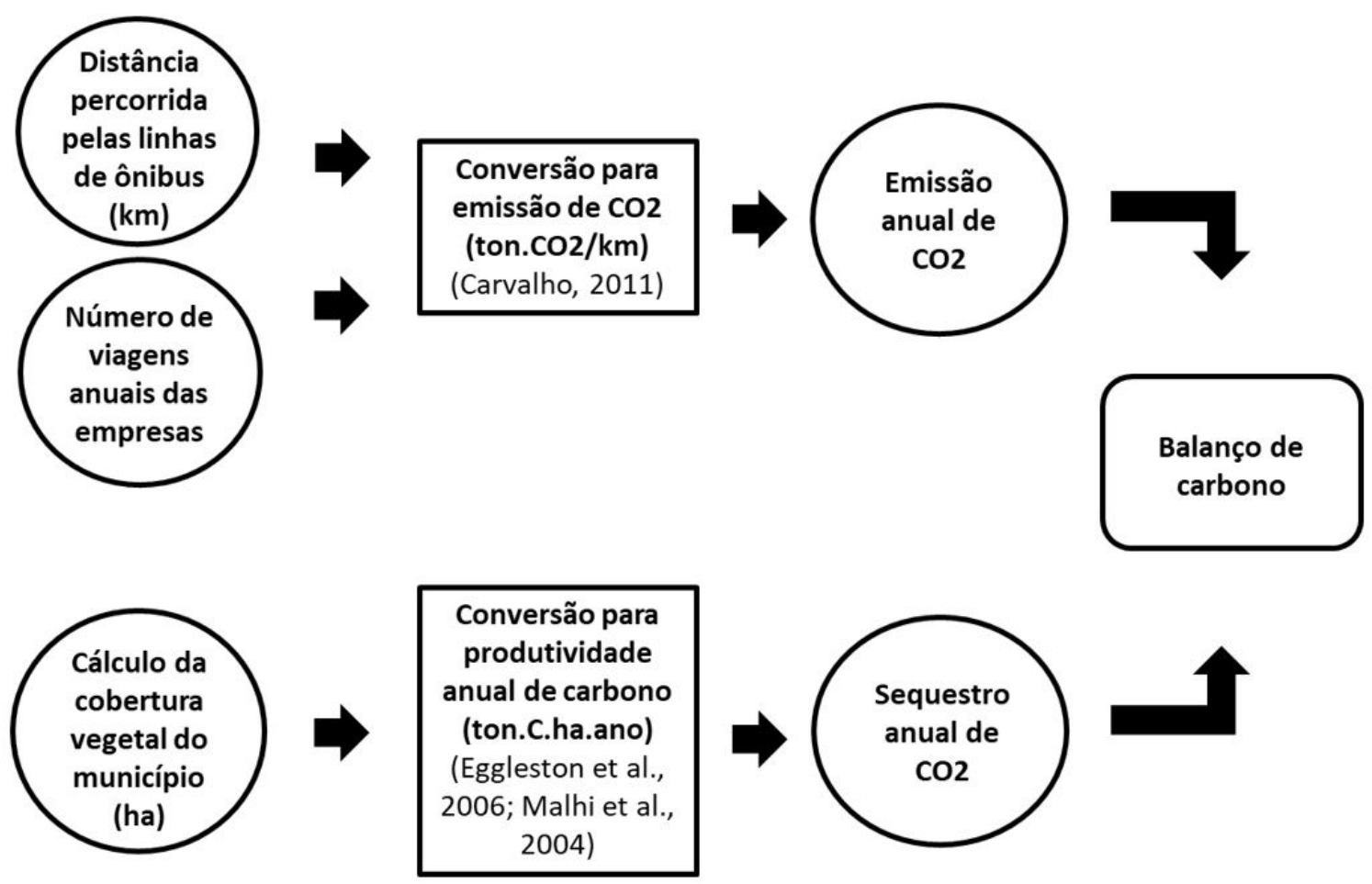

Figura 2 - Fluxograma de etapas para determinação do balanço de carbono. Fonte: Autores (2018). 
Quadro 3 - Descrição das fórmulas empregadas

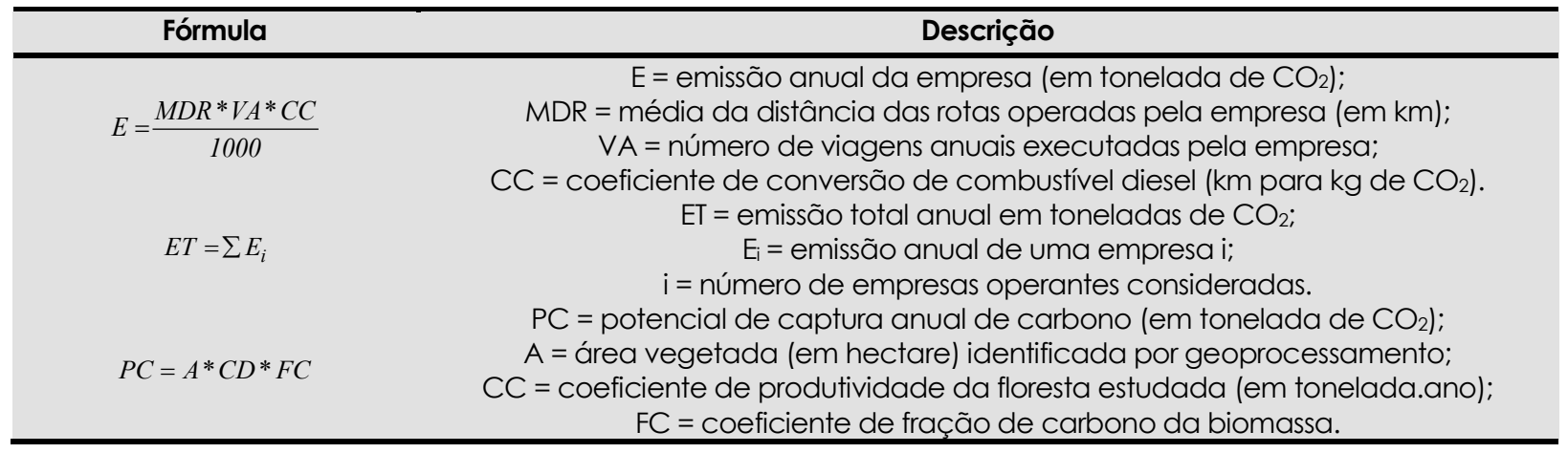

Fonte: Autores (2018).

A partir dos resultados obtidos no balanço final de carbono, dois cenários distintos (um de balanço positivo e outro negativo) podem ser alcançados (Quadro 4). Há um cenário de balanço negativo de carbono quando a captura anual de carbono for menor que as emissões anuais de $\mathrm{CO}_{2}$. Por sua vez, há um cenário positivo de balanço quando a emissão anual de $\mathrm{CO}_{2}$ for menor que o potencial de captura anual de $\mathrm{CO}_{2}$ (não havendo, portanto, parcelas de carbono remanescentes na atmosfera).

Quadro 4 - Cenários de balanço de carbono

\begin{tabular}{cl}
\hline Cenário & \multicolumn{1}{c}{ Descrição } \\
\hline & Potencial de captura anual de $\mathrm{CO}_{2}$ pela vegetação \\
Balanço negativo & menor que emissão anual de $\mathrm{CO}_{2}$ pelo transporte \\
& público, apresentando uma parcela de carbono não \\
& capturada pela vegetação. \\
& Emissão anual de $\mathrm{CO}_{2}$ pelo transporte público menor \\
& que potencial de captura anual de $\mathrm{CO}_{2}$ pela \\
Balanço positivo & vegetação, não havendo remanescentes de carbono \\
& não capturados pela vegetação. \\
\hline
\end{tabular}

Fonte: Autores (2018).

\section{Análise como instrumento de política pública}

Para a análise do balanço de carbono como um instrumento de política pública, realizou-se uma abordagem descritiva, com o objetivo de descrever as características de determinado fenômeno, estabelecendo a relação entre as variáveis (Gil, 2008). Com a pesquisa descritiva, é possível classificar, explicar e interpretar os fatos que ocorrem a partir dos objetivos propostos no estudo (Prodanov \& Freitas, 2013). A pesquisa bibliográfica foi realizada por meio de uma revisão de literatura que analisou estudos relevantes que tratam da temática de políticas públicas e cidades sustentáveis.

\section{Resultados e discussões}

A partir do cálculo das distâncias percorridas pelas 178 rotas de ônibus, identificou-se uma distância média de percurso de $32,42 \mathrm{~km}$. 0 trajeto de maior distância correspondeu a 70,9 km, enquanto o de menor distância equivaleu a 13,30 km. As rotas de maior distância de percurso foram as originadas em localidades mais afastadas do centro da cidade, com destaque para as oriundas dos distritos de Icoaraci, Bengui e Outeiro. Os valores encontrados estão apresentados no Quadro 5. 
Quadro 5 - Dados obtidos para as rotas das empresas de ônibus da RMB e a emissão anual de $\mathrm{CO}_{2}$ estimada

\begin{tabular}{|c|c|c|c|c|c|}
\hline Empresa & $\begin{array}{l}\text { Viagens anuais } \\
\text { contratadas }\end{array}$ & $\begin{array}{l}\mathbf{N}^{\circ} \text { de linhas } \\
\text { operadas }\end{array}$ & $\begin{array}{l}\text { Distância média } \\
\text { percorrida por } \\
\text { linhas operadas } \\
(\mathrm{km})\end{array}$ & $\begin{array}{l}\text { Distância anual } \\
\text { percorrida pela } \\
\text { frota }(\mathrm{km})\end{array}$ & $\begin{array}{l}\text { Emissão anual } \\
\text { de } \mathrm{CO}_{2} \text { (ton) }\end{array}$ \\
\hline Empresa A & 113.472 & 3 & 26,63 & $3.021 .759,36$ & $3.142,63$ \\
\hline Empresa B & 166.224 & 5 & 38,28 & 6.363.054,72 & $6.617,58$ \\
\hline Empresa C & 647.280 & 26 & 25,55 & $16.538 .004,00$ & $17.199,52$ \\
\hline Empresa D & 350.832 & 11 & 27,57 & $9.672 .438,24$ & $10.059,34$ \\
\hline Empresa E & 264.480 & 10 & 27,93 & $7.386 .926,40$ & $7.682,40$ \\
\hline Empresa B & 121.824 & 4 & 21,38 & $2.604 .597,12$ & $2.708,78$ \\
\hline Empresa F & 263.808 & 1 & 27,39 & $7.225 .701,12$ & $7.514,73$ \\
\hline Empresa G & 457.152 & 21 & 42,44 & $19.401 .530,88$ & $20.177,59$ \\
\hline Empresa $\mathrm{H}$ & 144.624 & 13 & 23,55 & $3.405 .895,20$ & $3.542,13$ \\
\hline Empresa I & 807.840 & 40 & 45,56 & $36.805 .190,40$ & $38.277,40$ \\
\hline Empresa J & 46.080 & 3 & 19,97 & $920.217,60$ & 957,03 \\
\hline Empresa K & 370.176 & 17 & 32,92 & $12.186 .193,92$ & $12.673,64$ \\
\hline Empresa L & 94.608 & 4 & 23,15 & $2.190 .175,20$ & $2.277,78$ \\
\hline Empresa M & 197.952 & 6 & 24,93 & $4.934 .943,36$ & $5.132,34$ \\
\hline Empresa N & 75.360 & 2 & 26,72 & $2.013 .619,20$ & $2.094,16$ \\
\hline Empresa O & 77.184 & 2 & 25,90 & $1.999 .065,60$ & $2.079,03$ \\
\hline Empresa P & 25.968 & 1 & 26,50 & $688.152,00$ & 715,68 \\
\hline Empresa Q & 25.152 & 3 & 54,80 & $1.378 .329,60$ & $1.433,46$ \\
\hline Empresa R & 38.688 & 3 & 34,43 & $1.332 .027,84$ & $1.385,31$ \\
\hline Empresa S & 69.360 & 8 & 16,90 & $1.172 .184,00$ & $1.219,07$ \\
\hline Total & 4.358 .064 & 183 & 592,50 & $141.240 .005,76$ & $146.889,61$ \\
\hline Média & 217.903 & 9 & 32,42 & 7.062 .000 & 7.344 \\
\hline Mínima & 25.152 & 1 & 13,30 & 688.152 & 716 \\
\hline Máxima & 807.840 & 40 & 70,9 & 36.805 .190 & 38.277 \\
\hline
\end{tabular}

Fonte: Autores (2018).

Em termos de contribuições gerais para as emissões de $\mathrm{CO}_{2}$, destaca-se que três empresas ( $\mathrm{C}$, $\mathrm{G}$ e I) corresponderam a, aproximadamente, $50 \%$ das emissões totais (Quadro 5). Essas empresas operaram uma quantidade superior de linhas de ônibus $(26,21$ e 40 linhas, respectivamente) e realizaram, consequentemente, um número de viagens anuais também elevado (647.280, 457.152 e 807.840 viagens, respectivamente). Entre elas, a empresa I destaca-se por, além de possuir os valores mais altos de linhas operadas e viagens realizadas, apresentar uma distância média de percurso acima da média - resultando, consequentemente, em uma distância anual total percorrida bem superior a outras empresas.

Considerando as contribuições de emissões de todas as empresas, obteve-se, portanto, um total de 146.889,61 toneladas de $\mathrm{CO}_{2}$ emitidas no ano de 2016. Ressalta-se que algumas linhas não eram de operação exclusiva de uma única empresa, o que gerou um número total de linhas operadas maior que 178.

Foram identificados $1.185,87$ ha $(4,49 \%)$ de vegetação rasteira, 5.495,32 ha $(20,78 \%)$ de hidrografia e 11.400,00 ha (43,12\%) de área impermeável. 0 Quadro 6 sintetiza os dados identificados na análise de classificação do solo.

Quadro 6 - Áreas identificadas por tipologia de classificação do solo

\begin{tabular}{cccc}
\hline Tipologia & $\mathbf{N}^{\circ}$ de polígonos & Área $(\mathbf{h a})$ & $\%$ \\
\hline Vegetação densa & 23.858 & $8.358,97$ & 31,61 \\
Vegetação rasteira & 51.843 & $1.185,87$ & 4,49 \\
Hidrografia & 430 & $5.495,32$ & 20,78 \\
Impermeável & 84.543 & 11.400 & 43,12 \\
Total & $\mathbf{1 6 0 . 6 7 4}$ & $\mathbf{2 6 . 4 4 0}$ & $\mathbf{1 0 0}$ \\
\hline
\end{tabular}

Fonte: Autores (2018).

Por sua vez, por meio também da análise de cobertura do solo (Figura 3), foi identificada uma área de vegetação densa de 8.358,97 hectares, correspondendo a 31,61\% da área territorial total considerada. Esses dados se assimilam a valores encontrados na literatura, como os estudos de Tavares et al. (2019) e 
Amaral et al. (2009), que encontraram, respectivamente, 31\% de remanescente de floresta nativa e 37\% de cobertura vegetal no território de Belém.

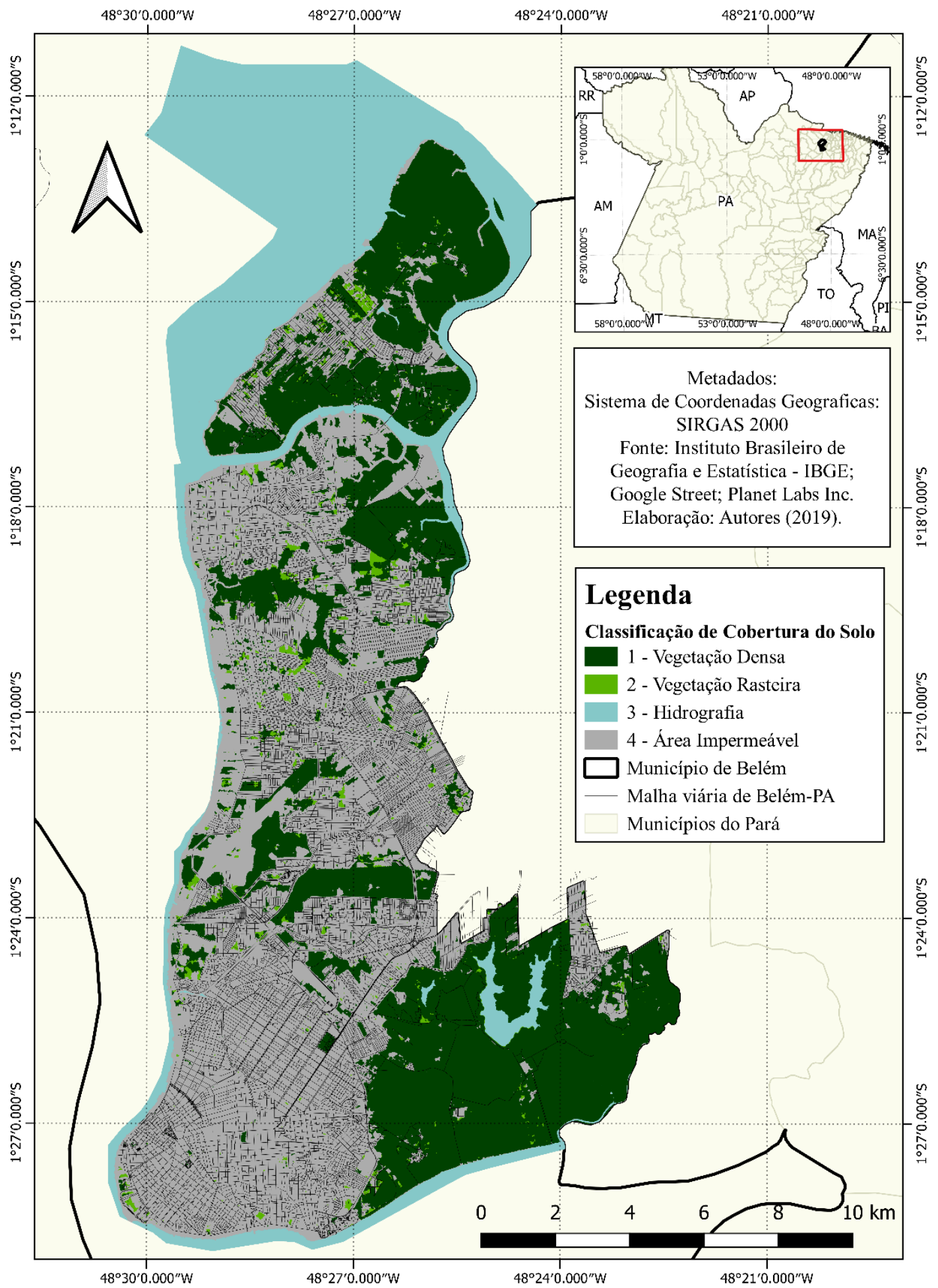

Figura 3 - Classificação semiautomática da área considerada no estudo. Fonte: Autores (2018).

Considerando a área de cobertura vegetal densa e aplicando-se a taxa de incremento de biomassa seca anual, obteve-se um valor de crescimento equivalente a 10.444,53 toneladas de matéria seca/hectare/ano. Dessa maneira, ao aplicar a taxa de conversão para porcentagem de carbono, identificou-se um potencial de captura de carbono equivalente a 5.117,82 toneladas/hectare/ano. 
Ressalta-se que esse valor faz referência apenas ao crescimento da parte superior da vegetação, não sendo englobado, portanto, o crescimento da biomassa abaixo do solo.

Consequentemente, equiparando os dados de emissão de $\mathrm{CO}_{2}$ com os de captura anual de carbono, verificou-se que a captura correspondeu a $3,48 \%$ do quantitativo de $\mathrm{CO}_{2}$ emitido. Há, portanto, um balanço negativo de carbono, com um saldo de $\mathrm{CO}_{2}$ não capturado pela vegetação equivalente a 141.771,78 toneladas (Figura 4). Ainda, apesar da diferença de ano entre as imagens de satélite e os dados de transporte público municipal, o município de Belém se constitui em uma área urbanizada consolidada, apresentando pequenas variações na sua cobertura vegetal entre períodos como esse.

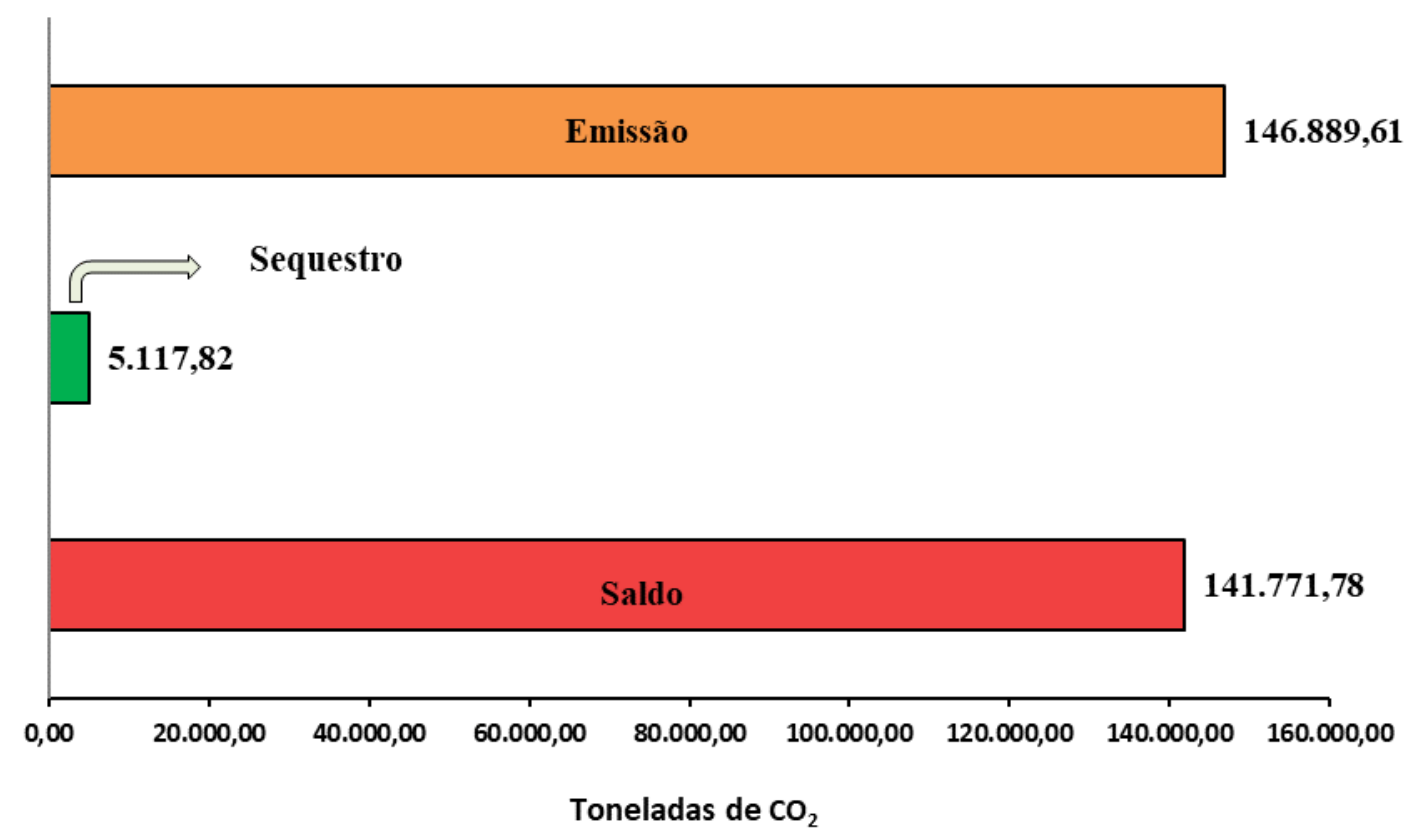

Figura 4 - Balanço geral de $\mathrm{CO}_{2}$. Fonte: Autores (2018).

Avaliando, portanto, o potencial de captura da vegetação em função do total de $\mathrm{CO}_{2}$ emitido pelo transporte público, constata-se que, por si, a captura não apresentou grande capacidade de mitigação dessas emissões. Nesse sentido, torna-se relevante fazer inferências sobre três principais questões: as emissões totais de $\mathrm{CO}_{2}$ no município, o potencial de ampliação da captura de carbono no município e a atuação integrada de políticas públicas.

No que tange às emissões globais do município, enfatiza-se que este estudo se limitou a calcular as emissões de apenas uma parcela da frota de veículos do município. Assim, há uma parcela significativa não contabilizada de emissões por parte dos 289.537 automóveis individuais existentes na cidade. Complementarmente, ressalta-se a existência de emissões por outros setores, como processos industriais e geração de resíduos sólidos, assim como verificado no Rio de Janeiro e em São Paulo (Alonso et al., 2010; Dodman, 2009). Dessa maneira, o déficit do balanço de carbono tende a crescer, mesmo que não tenha sido considerado o potencial de captura da vegetação abaixo do solo e áreas de grande importância em termos de cobertura vegetal, como as ilhas da cidade e o distrito de Mosqueiro.

Sobre a captura de carbono da vegetação do município, constatou-se que, geograficamente, a maior parte da vegetação se localizava concentrada em áreas periféricas e menos habitadas da cidade. Dessa forma, duas inferências principais são tomadas: há potencial para aumento da cobertura vegetal em áreas mais centrais da cidade; e as áreas centrais de baixa cobertura vegetal podem apresentar escassez de benefícios gerados pela vegetação, como redução de temperaturas superficiais e mitigação de cheias (Roy et al., 2012; Tyrväinen et al., 2014; Dimoudi \& Nikolopoulou, 2003). Assim, políticas de aumento de cobertura vegetal no município podem aumentar o potencial de captura de $\mathrm{CO}_{2}$ e a qualidade de vida na cidade.

No âmbito das políticas públicas, por sua vez, constatou-se a necessidade adoção de uma perspectiva multidisciplinar e de políticas integradas para alcançar valores mais substanciais na mitigação de 
emissões de $\mathrm{CO}_{2}$, visto que a captura, por si, representou valores modestos. Nesse sentido, exemplos bemsucedidos de programas adotados por diferentes cidades ao redor do mundo podem ser relevantes, como as que envolvem intervenções nas áreas de transporte e aumento de densidade urbana (Lee \& Erickson, 2017; Broto \& Bulkeley, 2013). Pode-se dar maior ênfase às políticas de transporte, como as melhorias no transporte coletivo e a restrição e o incentivo a transportes de baixo carbono, por causa dos contínuos aumentos na frota de veículos do município de Belém nos últimos anos (Rodrigues, 2013).

Complementarmente, os resultados obtidos, quando comparados com os valores identificados por Guo et al. (2017), aproximadamente $25 \%$ de potencial de captura de um total de 3.200 toneladas de $\mathrm{CO}_{2}$ em 2013, explicitam grandes diferenças quantitativas em relação a este estudo. Evidencia-se, portanto, a importância da seleção de metodologias para estudos de captura e balanço de carbono, como enfatizado por Fearnside (1994). Tal fator se torna ainda mais relevante por conta da correlação desses temas com a questão climática global, que requer precisão e atuação integrada entre diferentes países para o sucesso das intervenções.

\section{Considerações finais}

As análises realizadas permitiram a identificação de um potencial de captura de carbono pela vegetação municipal equivalente a 5.117,82 toneladas/ano e de uma emissão de $\mathrm{CO}_{2}$ pela frota de ônibus no município correspondente a 146.889,61 toneladas para o ano de 2016. Obteve-se, portanto, um balanço de carbono com saldo negativo, com 141.771,78 de $\mathrm{CO}_{2}$ não sequestrado. Nesse cenário, a captura correspondeu a $3,48 \%$ do quantitativo emitido.

Dessa maneira, considerando as emissões não contabilizadas por automóveis individuais no município, constata-se que o atual potencial de captura exercido pela vegetação urbana, por si, não foi tão substancial. Verificou-se, no entanto, a possibilidade de aumento desse potencial por meio de políticas públicas, as quais podem proporcionar melhorias na qualidade de vida em áreas menos arborizadas da cidade.

Para alcançar valores mais significativos na redução geral de emissões de $\mathrm{CO}_{2}$ no município, por sua vez, observou-se a importância da adoção de políticas ambientais integradas e de perspectivas multidisciplinares, tais como políticas de transporte e outras que afetem essa área em razão dos aumentos de frota de veículos que ocorrem em Belém. Dessa maneira, tornou-se evidente também a importância de estudos de balanços de carbono locais, assim como os de avaliação de políticas públicas, para o planejamento sustentável de cidades.

Para ampliar essas possibilidades de planejamento de políticas eficazes no município, constatou-se a necessidade de geração de subsídios científicos auxiliares sobre a questão climática. Nesse sentido, sugere-se a realização estudos que investiguem as emissões por automóveis individuais e de estudos que considerem de maneira integrada as emissões do sistema de transportes municipal. Ainda, há a necessidade de investigação de emissões de $\mathrm{CO}_{2}$ por outros setores, como as atividades industriais e por geração de resíduos sólidos na Região Metropolitana de Belém.

\section{Referências}

Alonso, M. F., Longo, M. K., Freitas, S. R., Fonseca, R. M., Marécal, V., Pirre, M., \& Klenner, G. L. (2010). An urban emissions inventory for South America and its application in numerical modeling of atmospheric chemical composition at local and regional scales. Atmospheric Environment, 44(39), 5072-5083.

http://dx.doi.org/10.1016/j.atmosenv.2010.09.013.

Amaral, D. D., Vieira, I. C. G., Almeida, S. S., Salomão, R. P., Silva, A. S. L., \& Jardim, M. A. G. (2009). Checklist da flora arbórea de remanescentes florestais da região metropolitana de Belém e valor histórico dos fragmentos, Pará, Brasil. Boletim do Museu Paraense Emílio Goeldi. Ciências Naturais, 4(3), 231-289. Recuperado em 14 de agosto de 2018, de http://scielo.iec.gov.br/scielo.php?script=sci_arttext\&pid=S1981-81142009000300002\&lng=pt\&tlng=pt 
Andrade, J. C. S., \& Costa, P. (2008). Mudança climática, protocolo de Kyoto e mercado de créditos de carbono: desafios à governança ambiental global. Organizações \& Sociedade, 15(45), 29-45.

http://dx.doi.org/10.1590/S1984-92302008000200002.

Belém. (2012). Anuário estatístico do município de Belém 2011. Belém: Secretaria Municipal de Coordenação Geral do Planejamento e Gestão. Recuperado em 14 de agosto de 2018, de http://www.belem.pa.gov.br/app/ANUARIO_2011/ANUARIO\%202011\%20COMPLETO.pdf

Brasil. Ministério da Ciência, Tecnologia, Inovações e Comunicações. Projeto de Estimativa de Desfloramento da Amazônia - PRODES. (2017). Monitoramento da floresta Amazônica Brasileira por satélite. São José dos Campos. Recuperado em 13 de agosto de 2018, de http://www.obt.inpe.br/OBT/assuntos/programas/amazonia/prodes

Broto, V. C., \& Bulkeley, H. (2013). A survey of urban climate change experiments in 100 cities. Global Environmental Change, 23(1), 92-102. http://dx.doi.org/10.1016/j.gloenvcha.2012.07.005. PMid:23805029.

Cardoso, A. C. D., \& Ventura, R. S., No. (2013). A evolução urbana de Belém: trajetória de ambiguidades e conflitos socioambientais. Cadernos Metrópole, 15(29).

Carvalho, C. H. R. (2011). Emissões relativas de poluentes do transporte motorizado de passageiros nos grandes centros urbanos brasileiros (Boletim regional, urbano e ambiental; No. 5). Brasília: IPEA. Recuperado em 14 de agosto de 2018, de http://repositorio.ipea.gov.br/bitstream/11058/5574/1/BRU_n05_emiss\%C3\%B5es.pdf

Carvalho, R. M., \& Szlafsztein, C. F. (2019). Urban vegetation loss and ecosystem services: the influence on climate regulation and noise and air pollution. Environmental Pollution, 245, 844-852.

http://dx.doi.org/10.1016/j.envpol.2018.10.114. PMid:30504036.

Coskun, D., Britto, D. T., \& Kronzucker, H. J. (2016). Nutrient constraints on terrestrial carbon fixation: the role of nitrogen. Journal of Plant Physiology, 203, 95-109. http://dx.doi.org/10.1016/j.jplph.2016.05.016.

PMid:27318532.

D’Agosto, M. A. (2015). Transporte, uso de energia e impactos ambientais: uma abordagem introdutória. Rio de Janeiro: Elsevier.

Dimoudi, A., \& Nikolopoulou, M. (2003). Vegetation in the urban environment: microclimatic analysis and benefits. Energy and Building, 35(1), 69-76. http://dx.doi.org/10.1016/S0378-7788(02)00081-6.

Dodman, D. (2009). Blaming cities for climate change? An analysis of urban greenhouse gas emissions inventories. Environment and Urbanization, 21(1), 185-201. http://dx.doi.org/10.1177/0956247809103016.

Eggleston, H. S., Buendia, L., Miwa, K., Ngara, T., \& Tanabe, K. (2006). 2006 IPCC guidelines for national greenhouse gas inventories. Japan: IGES.

Farr, D. (2011). Sustainable urbanism: urban design with nature. Hoboken: John Wiley \& Sons.

Fearnside, P. M. (1994). Biomassa das florestas Amazônicas brasileiras. In R. L. Bandeira, M. Reis, M. N. Borgonovi, \& S. Cedrola (Eds.), Emissão × seqüestro de CO2: uma nova oportunidade de negócios para o Brasil (pp. 95-124). Rio de Janeiro: Companhia Vale do Rio Doce.

Fearnside, P. M. (1997). Wood density for estimating forest biomass in Brazilian Amazonia. Forest Ecology and Management, 90(1), 59-87. http://dx.doi.org/10.1016/S0378-1127(96)03840-6.

Fearnside, P. M. (2009). A vulnerabilidade da floresta amazônica perante as mudanças climáticas. Oecologia Brasiliensis, 13(4), 609-618. http://dx.doi.org/10.4257/oeco.2009.1304.05.

Frey, K. (2009). Políticas públicas: um debate conceitual e reflexões referentes à prática da análise de políticas públicas no Brasil. Planejamento e Políticas Públicas, 21, 211-259. Recuperado em 14 de agosto de 2018, de http://www.ipea.gov.br/ppp/index.php/PPP/article/view/89

Gil, A. C. (2008). Como elaborar projetos de pesquisa (4. ed.). São Paulo: Atlas.

Grace, J., Lloyd, J., McIntyre, J., Miranda, A. C., Meir, P., Miranda, H. S., Nobre, C., Moncrieff, J., Massheder, J., Malhi, Y., Wright, I., \& Gash, J. (1995). Carbon dioxide uptake by an undisturbed tropical rain forest in southwest Amazonia, 1992 to 1993. Science, 270(5237), 778-780. http://dx.doi.org/10.1126/science.270.5237.778. 
Guo, R., Zhao, Y., Shi, Y., Li, F., Hu, J., \& Yang, H. (2017). Low carbon development and local sustainability from a carbon balance perspective. Resources, Conservation and Recycling, 122, 270-279.

http://dx.doi.org/10.1016/j.resconrec.2017.02.019.

Heilig, G. K. (2012). World urbanization prospects: the 2011 revision. New York: United Nations, Department of Economic and Social Affairs (DESA), Population Division, Population Estimates and Projections Section.

Higuchi, N., Chambers, J., Santos, J., Ribeiro, R. J., Pinto, A. C. M., Silva, R. P., Rocha, R. D. M., \& Tribuzy, E. S. (2004). Dinâmica e balanço do carbono da vegetação primária da Amazônia Central. Floresta, 34(3).

http://dx.doi.org/10.5380/rf.v34i3.2417.

Houghton, R. A., \& Woodwell, G. M. (1989). Global climate change. Scientific American, 260(4), 36-44. http://dx.doi.org/10.1038/scientificamerican0489-36. PMid:2781259.

Instituto Brasileiro de Geografia e Estatística - IBGE. (2010). Censo demográfico 2010. Rio de Janeiro: IBGE. Recuperado em 13 de agosto de 2018, de https://cidades.ibge.gov.br

Instituto Brasileiro de Geografia e Estatística - IBGE. (2012). Manual técnico da vegetação brasileira (2. ed.). Rio de Janeiro: IBGE.

Kennedy, C., Cuddihy, J., \& Engel-Yan, J. (2007). The changing metabolism of cities. Journal of Industrial Ecology, 11(2), 43-59. http://dx.doi.org/10.1162/jie.2007.1107.

Lee, C. M., \& Erickson, P. (2017). How does local economic development in cities affect global GHG emissions? Sustainable Cities and Society, 35, 626-636. http://dx.doi.org/10.1016/j.scs.2017.08.027.

Luyssaert, S., Schulze, E. D., Börner, A., Knohl, A., Hessenmöller, D., Law, B. E., Ciais, P., \& Grace, J. (2008). Oldgrowth forests as global carbon sinks. Nature, 455(7210), 213-215. http://dx.doi.org/10.1038/nature07276. PMid:18784722.

Malhi, Y., Baker, T. R., Phillips, O. L., Almeida, S., Alvarez, E., Arroyo, L., Chave, J., Czimczik, C. I., Fiore, A. D., Higuchi, N., Killeen, T. J., Laurance, S. G., Laurance, W. F., Lewis, S. L., Montoya, L. M. M., Monteagudo, A., Neill, D. A., Vargas, P. N., Patino, S., Pitman, N. C. A., Quesada, C. A., Salomao, R., Silva, J. N. M., Lezama, A. T., Martinez, R. V., Terborgh, J., Vinceti, B., \& Lloyd, J. (2004). The above ground coarse wood productivity of 104 Neotropical forest plots. Global Change Biology, 10(5), 563-591. http://dx.doi.org/10.1111/j.1529-8817.2003.00778.x.

Maricato, E. (2013). Brasil, cidades: alternativas para a crise urbana (7. ed.) Petrópolis: Vozes.

Montagnini, F., \& Nair, P. K. R. (2004). Carbon sequestration: an underexploited environmental benefit of agroforestry systems. Agroforestry Systems, 61(1), 281-295.

http://dx.doi.org/10.1023/B:AGF0.0000029005.92691.79.

Nobre, C. A. (2008). Mudanças climáticas e o Brasil: contextualização. Parcerias Estratégicas, 13(27), 7-18.

Recuperado em 13 de agosto de 2018, de

http://seer.cgee.org.br/index.php/parcerias_estrategicas/article/viewFile/326/320

Nowak, D. J., \& Crane, D. E. (2002). Carbon storage and sequestration by urban trees in the USA. Environmental Pollution, 116(3), 381-389. http://dx.doi.org/10.1016/S0269-7491(01)00214-7. PMid:11822716.

Ohtsuka, T., Mo, W., Satomura, T., Inatomi, M., \& Koizumi, H. (2007). Biometric based carbon flux measurements and Net Ecosystem Production (NEP) in a temperate deciduous broad-leaved forest beneath a flux tower. Ecosystems, 10(2), 324-334. http://dx.doi.org/10.1007/s10021-007-9017-z.

Pachauri, R. K., \& Meyer, L. A. (2014). Climate change 2014: synthesis report. Contribution of Working Groups I, II and III to the fifth assessment report of the Intergovernmental Panel on Climate Change. Genebra: IPCC.

Pacheco, M. R. P. S., \& Helene, M. E. M. (1990). Atmosfera, fluxos de carbono e fertilização por $\mathrm{CO}_{2}$. Estudos Avançados, 4(9), 204-220. http://dx.doi.org/10.1590/S0103-40141990000200010.

Phillips, O. L., Malhi, Y., Higuchi, N., Laurance, W. F., Núñez, P. V., Vásquez, R. M., Laurance, S. G., Ferreira, L. V., Stern, V., Brown, S., \& Grace, J. (1998). Changes in the carbon balance of tropical forests: evidence from long-term plots. Science, 282(5388), 439-442. http://dx.doi.org/10.1126/science.282.5388.439. PMid:9774263. 
Poorter, L., van der Sande, M. T., Thompson, J., Arets, E. J. M. M., Alarcón, A., Álvarez-Sánchez, J., Ascarrunz, N., Balvanera, P., Barajas-Guzmán, G., Boit, A., Bongers, F., Carvalho, F. A., Casanoves, F., Cornejo-Tenorio, G., Costa, F. R. C., Castilho, C. V., Duivenvoorden, J. F., Dutrieux, L. P., Enquist, B. J., Fernández-Méndez, F., Finegan, B., Gormley, L. H. L., Healey, J. R., Hoosbeek, M. R., Ibarra-Manríquez, G., Junqueira, A. B., Levis, C., Licona, J. C., Lisboa, L. S., Magnusson, W. E., Martínez-Ramos, M., Martínez-Yrizar, A., Martorano, L. G., Maskell, L. C., Mazzei, L., Meave, J. A., Mora, F., Muñoz, R., Nytch, C., Pansonato, M. P., Parr, T. W., Paz, H., Pérez-García, E. A., Rentería, L. Y., RodríguezVelazquez, J., Rozendaal, D. M. A., Ruschel, A. R., Sakschewski, B., Salgado-Negret, B., Schietti, J., Simões, M., Sinclair, F. L., Souza, P. F., Souza, F. C., Stropp, J., ter Steege, H., Swenson, N. G., Thonicke, K., Toledo, M., Uriarte, M., van der Hout, P., Walker, P., Zamora, N., \& Peña-Claros, M. (2015). Diversity enhances carbon storage in tropical forests. Global Ecology and Biogeography, 24(11), 1314-1328. http://dx.doi.org/10.1111/geb.12364.

Prodanov, C. C., \& Freitas, E. C. (2013). Metodologia do trabalho científico: métodos e técnicas da pesquisa e do trabalho acadêmico (2. ed.). Novo Hamburgo: Feevale.

Rivero, S., Almeida, O., Ávila, S., \& Oliveira, W. (2009). Pecuária e desmatamento: uma análise das principais causas diretas do desmatamento na Amazônia. Nova Economia, 19(1), 41-66. http://dx.doi.org/10.1590/S010363512009000100003.

Rodrigues, J. M. (2013). Evolução da frota de automóveis e motos no Brasil 2001-2012: relatório 2013. Rio de Janeiro: Institutos Nacionais de Ciência e Tecnologia.

Rosa, I. M. D., Ahmed, S. E., \& Ewers, R. M. (2014). The transparency, reliability and utility of tropical rainforest land-use and land-cover change models. Global Change Biology, 20(6), 1707-1722.

http://dx.doi.org/10.1111/gcb.12523. PMid:24399778.

Roy, S., Byrne, J., \& Pickering, C. (2012). A systematic quantitative review of urban tree benefits, costs, and assessment methods across cities in different climatic zones. Urban Forestry \& Urban Greening, 11(4), 351-363. http://dx.doi.org/10.1016/j.ufug.2012.06.006.

Rozenstein, O., \& Karnieli, A. (2011). Comparison of methods for land-use classification incorporating remote sensing and GIS inputs. Applied Geography (Sevenoaks, England), 31(2), 533-544.

http://dx.doi.org/10.1016/j.apgeog.2010.11.006.

Santos, M. (2005). A urbanização brasileira. São Paulo: EDUSP.

Satterthwaite, D. (2008). Cities' contribution to global warming: notes on the allocation of greenhouse gas emissions. Environment and Urbanization, 20(2), 539-549. http://dx.doi.org/10.1177/0956247808096127.

Silveira, P., Koehler, H. S., Sanquetta, C. R., \& Arce, J. E. (2008). 0 estado da arte na estimativa de biomassa e carbono em formações florestais. Floresta, 38(1). http://dx.doi.org/10.5380/rf.v38i1.11038.

Su, M., Zheng, Y., Yin, X., Zhang, M., Wei, X., Chang, X., \& Qin, Y. (2016). Practice of low-carbon city in china: the status quo and prospect. Energy Procedia, 88, 44-51. http://dx.doi.org/10.1016/j.egypro.2016.06.014.

Tan, S., Yang, J., Yan, J., Lee, C., Hashim, H., \& Chen, B. (2017). A holistic low carbon city indicator framework for sustainable development. Applied Energy, 185, 1919-1930. http://dx.doi.org/10.1016/j.apenergy.2016.03.041.

Tavares, P. A., Beltrão, N. E. S., Guimarães, U. S., \& Teodoro, A. C. (2019). Integration of Sentinel-1 and Sentinel-2 for Classification and LULC Mapping in the Urban Area of Belém, Eastern Brazilian Amazon. Sensors, 19(5), 1-20. http://dx.doi.org/10.3390/s19051140. PMid:30845748.

Tyrväinen, L., Ojala, A., Korpela, K., Lanki, T., Tsunetsugu, Y., \& Kagawa, T. (2014). The influence of urban green environments on stress relief measures: a field experiment. Journal of Environmental Psychology, 38, 1-9. http://dx.doi.org/10.1016/j.jenvp.2013.12.005.

Vale, R., \& Balieiro, C. P. P. (2013). Diagnóstico ambiental no perímetro urbano do município de Marabá, estado do Pará, utilizando imagens de alta resolução. In Anais do XVI Simpósio Brasileiro de Sensoriamento Remoto (SBSR) (pp. 1061-1068). Foz do Iguaçu: INPE. 
Van Oudenhoven, A. P. E., Aukes, E., Bontje, L. E., Vikolainen, V., Van Bodegom, P. M., \& Slinger, J. H. (2018). 'Mind the Gap' between ecosystem services classification and strategic decision making. Ecosystem Services, 33, 77-88. http://dx.doi.org/10.1016/j.ecoser.2018.09.003.

Weisz, H., \& Steinberger, J. K. (2010). Reducing energy and material flows in cities. Current Opinion in Environmental Sustainability, 2(3), 185-192. http://dx.doi.org/10.1016/j.cosust.2010.05.010.

West, T. O., \& Marland, G. (2002). Net carbon flux from agricultural ecosystems: methodology for full carbon cycle analyses. Environmental Pollution, 116(3), 439-444. http://dx.doi.org/10.1016/S0269-7491(01)00221-4. PMid:11822723.

Woodbury, P. B., Smith, J. E., \& Heath, L. S. (2007). Carbon sequestration in the US forest sector from 1990 to 2010. Forest Ecology and Management, 241(1), 14-27. http://dx.doi.org/10.1016/j.foreco.2006.12.008.

Editor: Harry Bollmann

Recebido: Mar. 11, 2019

Aprovado: Mar. 19, 2019 